\title{
Il ruolo delle zone umide della Regione Lombardia durante la migrazione dei limicoli: un'analisi per il genere Tringa ricavata dal portale www.ornitho.it
}

\author{
Fabio Saporetti
}

\begin{abstract}
Riassunto - Nel 2009 l'avvio del portale www.ornitho.it ha giocato un ruolo fondamentale nella diffusione in tempo reale dell'informazione ornitologica relativa ad abbondanza, distribuzione geografica e fenologia dell'avifauna. Per molte specie il vasto database di ornitho. it permette di incrementare ulteriormente le conoscenze relative alla fenologia della migrazione, con aggiornamenti giornalieri derivati dalla partecipazione condivisa dei numerosi utenti. Ho analizzato i dati raccolti per il genere Tringa nel periodo 2010-2014, con un totale di 5.590 record, univoci e validati, inerenti la migrazione primaverile ed autunnale. L'analisi ha dimostrato l'importanza dell'area risicola lombarda, estesa per oltre 90.000 ettari prevalentemente in provincia di Pavia, per la migrazione primaverile, supportando i gruppi più numerosi delle specie del genere Tringa; altri ambienti, quali le cave rinaturalizzate, le vasche di spagliamento e raccolta delle acque, i ripristini ambientali, risultano altrettanto validi per il mantenimento della diversità dei Limicoli sebbene, assieme alle poche zone umide naturali, ospitino numeri decisamente inferiori di individui. La specie più abbondante è il Piro piro boschereccio con gruppi che raggiungono i 600 individui, seguito da Totano moro e Pantana. Il Piro piro culbianco è la specie più comune e quella che rivela la più ampia distribuzione geografica, con individui che raggiungono, durante la migrazione autunnale, le maggiori altitudini, a quote superiori ai 2.600 metri s.l.m. in provincia di Sondrio. La Pettegola è relativamente rara, generalmente presente a quote inferiori ai 200 metri, in provincia di Pavia e Milano; l'Albastrello si conferma una specie rara, con solo 68 record nel quinquennio.
\end{abstract}

Parole chiave: genere Tringa, fenologia, abbondanza, distribuzione geografica, Lombardia.

\begin{abstract}
The role of wetlands of the Lombardy Region during wader migration: an analysis of the genus Tringa, obtained through the portal www.ornitho.it.

In Italy, the launch of the online portal www.ornitho.it in 2009 has played a key role in advancing our knowledge about the abundance, geographical distribution and phenology of birds, and has greatly improved everyday ornithological issues. Indeed, the extensive participation by many observers has provided an opportunity to obtain new insight into the phenology of many species of migratory birds, such as Tringa sp. In this paper, I have analysed the data gathered between 2010 and 2014 on the genus Tringa in Lombardy, amounting to a total of
\end{abstract}

Gruppo Insubrico di Ornitologia Onlus, c/o Civico Museo Insubrico di Storia Naturale di Clivio e Induno Olona, Via Manzoni 21, 21050 Clivio (VA), Italia.

E-mail: saporettif@gmail.com

\section{C) 2017 Fabio Saporetti}

Received: 4 March 2016

Accepted for publication: 19 July 2017
5,590 validated records for spring and autumn migrations. The analysis revealed that the rice-farming area of S-W Lombardy - which covers more than 90,000 hectares located mainly in the province of Pavia represents an invaluable habitat during the spring migration by supporting the bulk of migrating waders. Other man-made habitats, such as restored sand and gravel pits and water-collection tanks, are of similar importance for the diversity of the wader community, but, together with the few natural wetlands, support much lower numbers of individuals. The wood sandpiper is the most abundant species, with groups reaching 600 individuals, followed by the spotted redshank and the greenshank. The green sandpiper is the most common and widespread species, with a low number of individuals reaching the highest altitudes (above 2,600 $\mathrm{m}$ a.s.l. in the province of Sondrio) during the autumn migration. The common redshank is relatively rare, generally distributed around 200 $\mathrm{m}$ a.s.l. in the provinces of Pavia and Milan, while the marsh sandpiper is definitely rare, with only 68 records in the five-year period analysed.

Key words: genus Tringa, phenology, abundance, geographical distribution, wetlands, Lombardy.

\section{INTRODUZIONE}

In Italia le sei specie del genere Tringa nidificanti nella regione paleartica sono migratori regolari di doppio passo (Spina \& Volponi, 2008), svernanti regolari (T. ochropus, T. erythropus, T. totanus, T. nebularia) od irregolari (T. stagnatilis e T. glareola; Brichetti \& Fracasso, 2004). La Pettegola, parzialmente sedentaria, è anche specie nidificante nelle lagune costiere dell'alto Adriatico, con coppie sparse nelle lagune salmastre della Toscana, Sardegna, Puglia e Sicilia (Brichetti \& Fracasso, 2004): nella parte occidentale della valle del Po la prima coppia nidificante nelle risaie piemontesi è stata rinvenuta nel 1994 (Della Toffola et al. 2003) e da allora una piccola popolazione ha nidificato fino al 2010 (Alessandria et al, 2011). In Italia nord-occidentale la conoscenza della fenologia della migrazione, della distribuzione geografica e dello svernamento dei limicoli, erano noti per il Piemonte, ad opera del Gruppo Piemontese Studi Ornitologici (GPSO), con le prime pubblicazioni risalenti agli anni '80 (Mingozzi, 1982), mentre per la Lombardia dati occasionali erano disponibili su "Natura Bresciana" per la Provincia di Brescia. Sempre negli anni ' 80 in Italia ebbero inizio i censimenti IWC (Barbieri, 1988) che fornirono le prime indicazioni sulle specie svernanti: successivamente, nel periodo 1986-1992, in Piemonte e Lombardia iniziarono i rilevamenti per l'elaborazione degli atlanti delle specie svernanti (Fornasari et al., 1992; Cucco et al, 1996) che, 
per i taxa considerati, produssero due carte di distribuzione relative a Piro piro culbianco e Pantana, comprendenti i mesi di dicembre e gennaio per il Piemonte, ed il periodo metà novembre - febbraio per la Lombardia. Nel 2007 Pillitteri Rosa et al. pubblicarono i risultati del monitoraggio biennale (2006-2007) relativo alla fenologia delle specie di interesse venatorio, comprendenti quindi Pavoncella e Beccaccino, relativamente a 17 zone umide della Lombardia occidentale. Al di fuori della letteratura citata, congiuntamente agli ormai regolari rapporti dei censimenti IWC del mese di gennaio, editi dal dipartimento di Eco-etologia dell'Università di Pavia (ad es. Longoni \& Fasola, 2012, 2013; Longoni et al., 2014), non esistono pubblicazioni a livello regionale concernenti la fenologia, la distribuzione e l'abbondanza dei Limicoli che transitano sul territorio lombardo. Solo alcune pubblicazioni a livello provinciale o locale, hanno parzialmente preso in esame l'argomento (Cairo et al., 2001; Tosi \& Zilio, 2002; Bordignon \& Corti, 2003; Baietto, 2005; Baietto \& Bottoni, 2005; Pirotta et al., 2009; Casale, 2015). In questo lavoro esamino i dati raccolti nel quinquennio 2010-2014 nel portale www.ornitho.it, analizzando la fenologia della migrazione, la distribuzione geografica e l'abbondanza delle 6 specie.

\section{MATERIALI E METODI}

Ai fini di questo lavoro ho presentato richiesta al Gruppo di Conduzione, fornendo le principali linee di sviluppo dell'analisi: ho ricevuto i record del quinquennio (con l'esclusione di quelli protetti) sotto forma di fogli Excel, comprendenti i 51 campi dei dati immagazzinati: per questa analisi ne ho utilizzati 12, elencati in Tab. 1. Ornitho. it è basato sulla griglia cartografica UTM con particelle di $10 \times 10 \mathrm{~km}$, ulteriormente suddivise in 100 celle di $1 \times 1$ $\mathrm{km}$; ogni osservazione può essere attribuita ad una cella, ad una località (ID località e località) od al centroide del comune.

Tab. 1 - Elenco delle 12 variabili utilizzate per l'analisi dei dati / List of the 12 variables used for data analysis.

\begin{tabular}{|c|c|}
\hline N. & Variabile \\
\hline 1 & nome della specie \\
\hline 2 & data \\
\hline 3 & anno \\
\hline 4 & giornata dell'anno \\
\hline 5 & pentade \\
\hline 6 & cella $1 \times 1 \mathrm{~km}$ \\
\hline 7 & località \\
\hline 8 & comune \\
\hline 9 & provincia \\
\hline 10 & numero degli individui \\
\hline 11 & altitudine \\
\hline 12 & nome dell'osservatore \\
\hline
\end{tabular}

Per illustrare la distribuzione geografica delle specie, raggruppando i dati per ambiti amministrativi comunali e provinciali, ho impiegato ArcView 9.0 (ESRI, Inc.; Redlands, CA, USA) utilizzando il database di uso del suolo Dusaf 4 della Regione Lombardia (www.geoportale. regione.lombardia.it), utilizzando il valore massimo registrato nel quinquennio in ogni comune. Il periodo della migrazione è stato suddiviso secondo Berthold (1973) in primavera (pentadi 1-34) ed autunno (pentadi (35-73): il numero massimo per pentade per ogni specie è stato ricavato assegnando ad ogni pentade, per ogni anno, il conteggio maggiore disponibile per ogni comune. L'analisi del numero di individui è stata condotta impiegando, per ogni anno, un dato per giorno per singola località o cella di $1 \times 1 \mathrm{~km}$ : nel caso di conteggi multipli riferiti alla stessa cella o località è stato utilizzato un solo conteggio selezionando, se necessario, il valore più elevato. Nel caso l'osservatore abbia utilizzato il GPS per inserire più conteggi nella stessa cella $1 \times 1 \mathrm{~km}$, riportando la localizzazione precisa, sono invece stati considerati tutti i conteggi immessi. Per due località con doppio codice identificativo (8.365 Pian di Spagna e 10.045 Pian di Spagna, zona umida IWC; 8.236 Parco delle Folaghe e 9.797 Parco Sovracomunale Le Folaghe, zona umida IWC) i dati sono stati considerati come facenti parte della medesima località. Ho utilizzato test parametrici (coefficiente di correlazione) e non (Mann-Whitney U test, Kruskal-Wallis test), in relazione alla normalità o meno dei dati, tenendo presente che i conteggi degli individui sono in generale fortemente asimmetrici; i test sono sempre bidirezionali. Ho usato i programmi VassarStats (www.vassarstats.com) e Past v. 3.06 (Hammer et al., 2001).

\section{RISULTATI}

Nel quinquennio sono stati raccolti complessivamente 6.756 record riferiti alle 6 specie: di questo totale, dopo l'eliminazione dei dati doppi o multipli riferiti alla stessa località, ne sono stati utilizzati 5590, definiti come numero corretto dei record, ripartiti secondo la suddivisione indicata in Tab. 2; la percentuale di dati eliminati varia dal $16.0 \%$ per 1'Albastrello al $22.2 \%$ per la Pettegola.

Il Piro piro culbianco risulta essere la specie con il maggior numero di record utili (1.984), seguito da Piro piro boschereccio (1.547) e dalla Pantana (1.202): queste 3 specie raccolgono complessivamente $1{ }^{\prime} 84,7 \%$ dei dati. Il Totano moro e la Pettegola, rispettivamente con 519 e 270 dati, presentano numeri molto inferiori, mentre l'Albastrello risulta essere una specie rara con solo 68 osservazioni nel quinquennio $(1,2 \%)$. La ripartizione geografica dei record totali interessa prevalentemente il settore sud-occidentale della regione, caratterizzato dalla diffusa presenza delle risaie, importante surrogato delle zone umide, in cui la provincia di Pavia rappresenta il centro di gravità del flusso migratorio, con ben il $73,8 \%$ dei dati raccolti; le altre province concorrono con percentuali di gran lunga inferiori, variando dal $6,7 \%$ della provincia di Milano allo $0,3 \%$ della provincia di Como. 
Tab. 2 - Numero totale e corretto dei record suddivisi per specie, periodo primaverile ed autunnale / Total and corrected number of records for each species in spring and autumn.

\begin{tabular}{|l|c|c|c|c|c|}
\hline Specie & $\begin{array}{c}\text { Numero totale } \\
\text { record }\end{array}$ & $\begin{array}{c}\text { Numero corretto } \\
\text { record }\end{array}$ & $\begin{array}{c}\text { \% eliminati } \\
\text { sul totale }\end{array}$ & Primavera & Autunno \\
\hline Tringa ochropus & 2377 & 1984 & 16.5 & 1197 & 787 \\
\hline Tringa glareola boschereccio & 1857 & 1547 & 16.7 & 962 & 585 \\
\hline Tringa nebularia & 1438 & 1202 & 16.4 & 828 & 374 \\
\hline Tringa erythropus & 656 & 519 & 20.9 & 404 & 115 \\
\hline Tringa totanus & 347 & 270 & 22.2 & 226 & 44 \\
\hline Tringa stagnatilis & 81 & 68 & 16.0 & 47 & 21 \\
\hline Totale & 6756 & 5590 & & 3664 & 1926 \\
\hline
\end{tabular}

\section{Piro piro culbianco}

Dopo il primo biennio, Il numero di record mostra un incremento costante a partire dal 2012, sia nel periodo primaverile (numero massimo nel 2014 con 403) che in quello autunnale (numero massimo nel 2014 con 240; Fig.

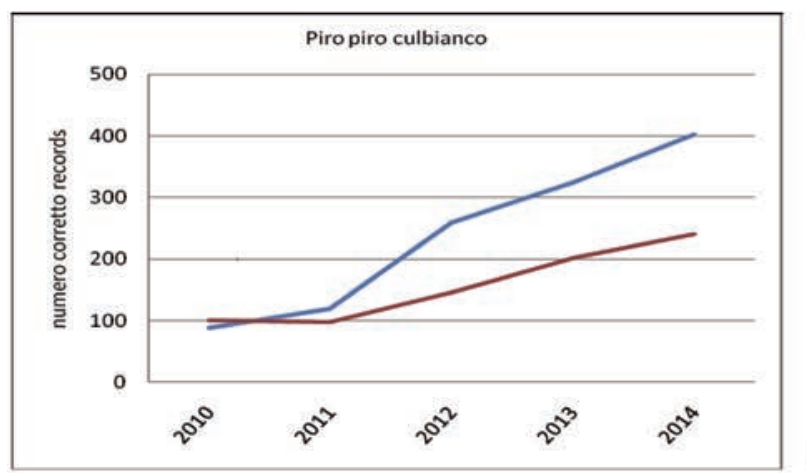

A
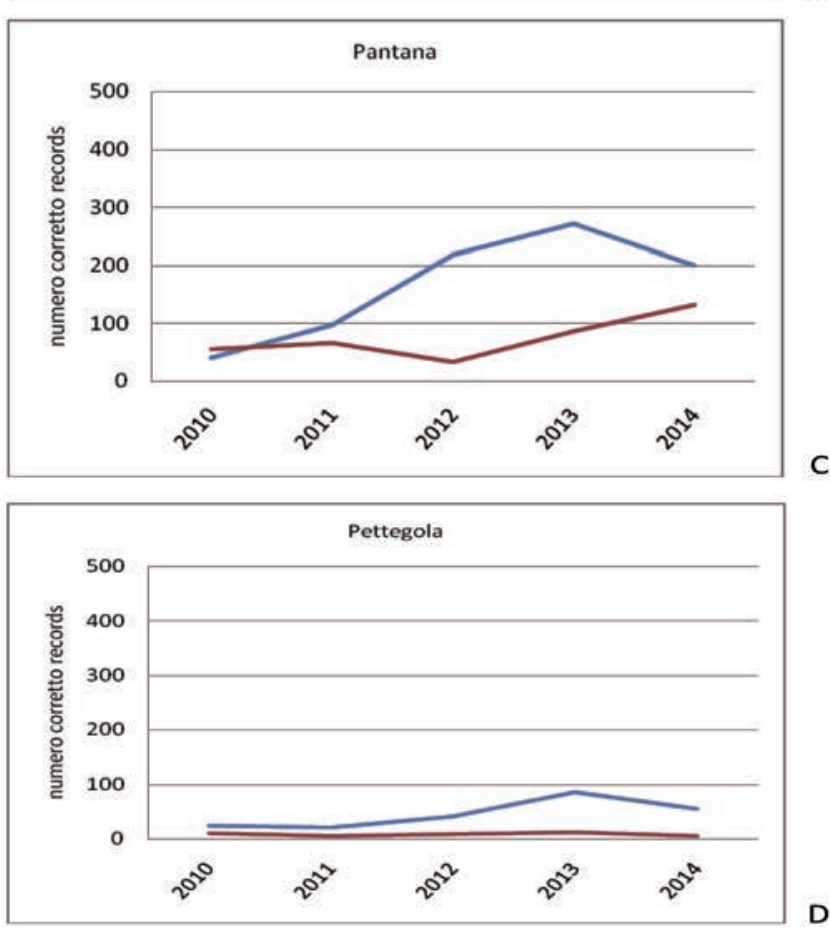

1A). Specie presente tutto l'anno, nel quinquennio 20102014 il flusso migratorio primaverile inizia alla pentade 14 (7-11 marzo), con il picco coincidente con la pentade 17 (22-26 marzo, Fig. 2A), corrispondente ad un massimo cumulativo di 147 individui; il minimo si registra in coin-
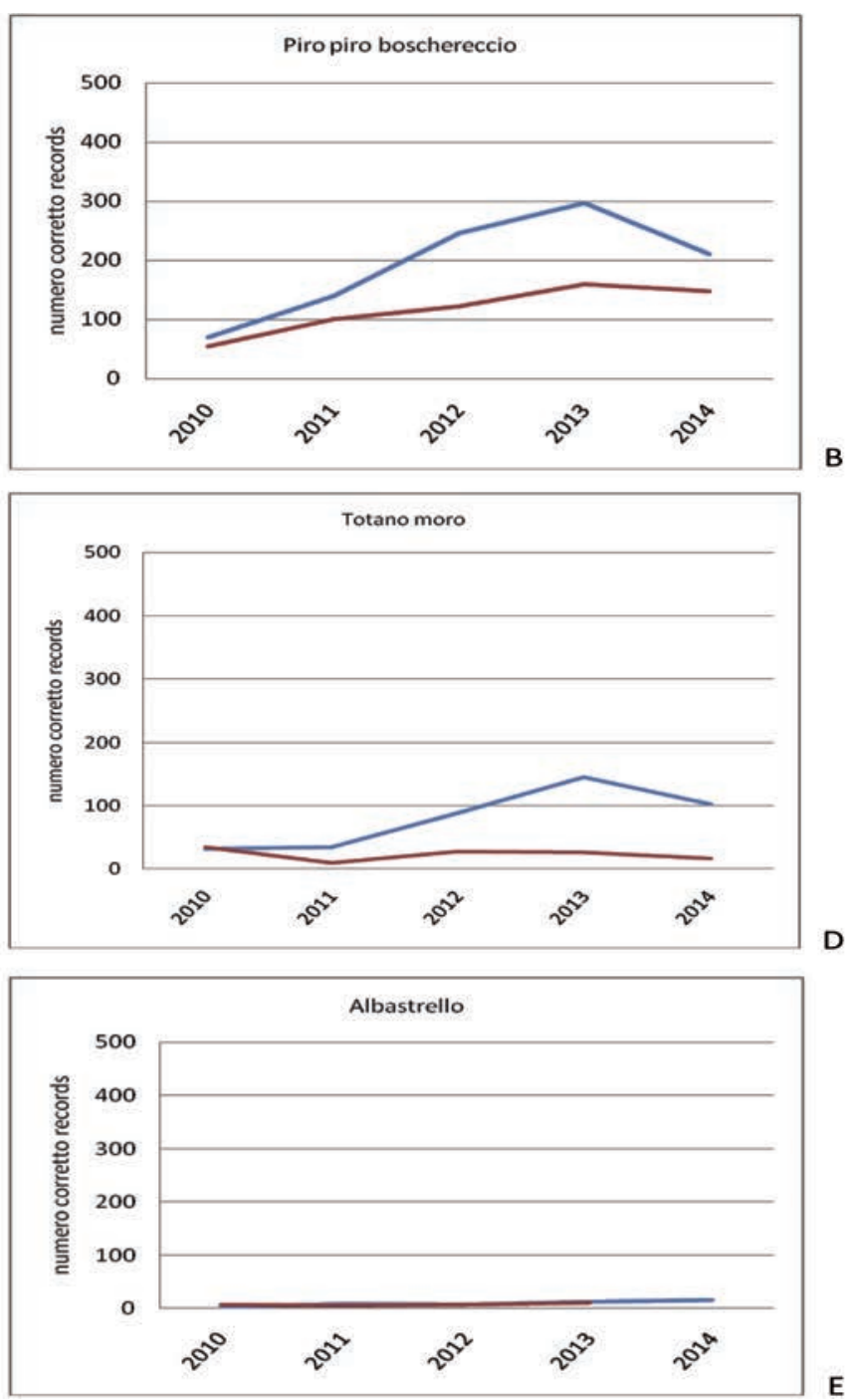

Fig. 1 - Numero corretto di records del quinquennio per le 6 specie. / The correct five-year records for the six species. 

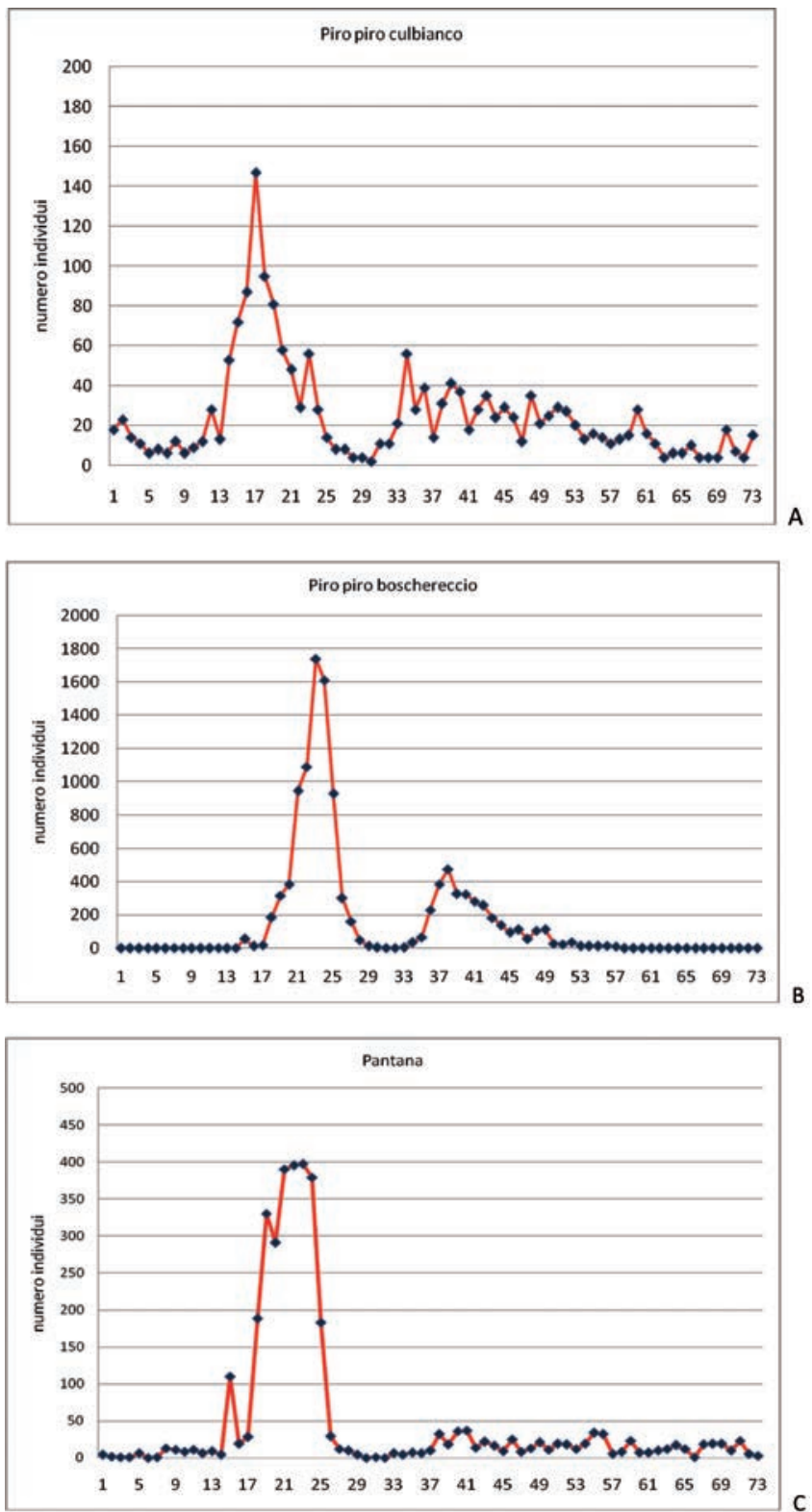

Fig. 2 - Numero massimo di individui per pentade nel quinquennio 2010-2014. / Maximum number of individuals for pentadas in the fiveyear period 2010-2014.

cidenza della pentade 29 (21-25 maggio). Il $1^{\circ}$ quartile varia dalla giornata 66 (7 marzo 2010) alla giornata 49 del 18 febbraio 2014, con una costante tendenza ad anticipare il passaggio dei primi contingenti, statisticamente significativa $(\mathrm{r}=-0,9559 ; \mathrm{t}=-5,64, \mathrm{P}=0,011)$; il terzo quartile corrisponde in entrambi gli anni alla giornata 106 (16 aprile; Fig. 3A); il valore mediano non presenta variazioni significative (Kruskal-Wallis $=0,2603, \mathrm{P}=0,9921$ ). La migrazione autunnale presenta un picco relativo alla pentade 34 (15-19 giugno; 56 individui), ma l'incremento numerico è già registrabile a partire dalla pentade 31 ( 31 maggio-4 giugno), per poi calare molto gradualmente nel corso del periodo estivo ed autunnale. La presenza del Piro piro culbianco è registrata in modo costante anche per il mese di dicembre (pentadi da 68 a73), ed il numero dei record aumenta dai 6 del 2010 (numero massimo di 2 individui)
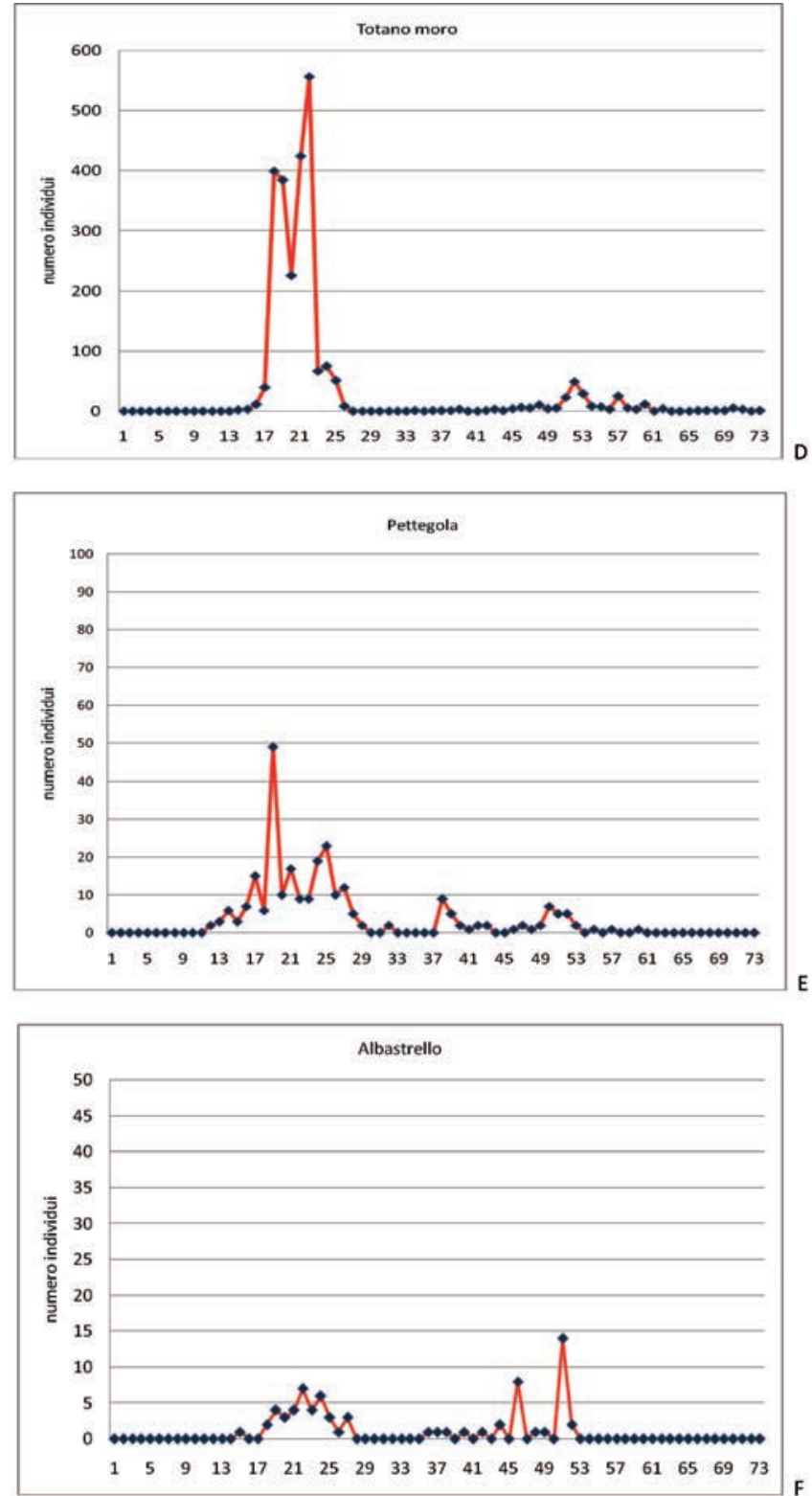

Fig. 3 - Numero massimo di individui per pentade nel quinquennio 2010-2014. / Maximum number of individuals for pentadas in the fiveyear period 2010-2014.

ai 27 del 2014, con un l'osservazione di un gruppo di 10 individui lungo il fiume Mincio, in provincia di Mantova (Mantovani C.). In primavera il Piro piro culbianco è presente con individui isolati, in coppia od in gruppi numericamente molto piccoli, generalmente inferiori a 10 individui: il valore mediano di individui conteggiati varia da 1 a 2 (Tab. 3) con valore massimo di 80 individui, rilevati il 22 marzo 2014 (Ilahiane L. \& Janni O.) alla Cassinazza di Baselica, in comune di Giussago (PV). In periodo autunnale i valori mediani sono pressoché uguali ai valori primaverili, con valori massimi (25 individui) rilevati nella medesima località il 13 luglio 2013 (Ilahiane L.): la differenza tra le mediane dei valori massimi primaverili e autunnali non è infatti statisticamente significativa (Mann-Whitney U test, $\mathrm{z}=1,46 ; \mathrm{P}=0,1443$ ). La distribuzione geografica della specie interessa tutte le province 
Tab. 3 - Numero dei record, dimensione dei conteggi (mediana e valore massimo) di Piro piro culbianco nel quinquennio 2010-2014. / Number of records and count results (median and maximum value) for the Green Sandpiper in the period 2010-2014.

\begin{tabular}{|l|c|c|c|c|c|}
\hline & $\mathbf{2 0 1 0}$ & $\mathbf{2 0 1 1}$ & $\mathbf{2 0 1 2}$ & $\mathbf{2 0 1 3}$ & $\mathbf{2 0 1 4}$ \\
\hline \multicolumn{7}{|c|}{ Primavera/Spring } \\
\hline Record & 89 & 120 & 260 & 325 & 403 \\
\hline Mediana conteggio & 2 & 1 & 2 & 2 & 1 \\
\hline SE & 0,35 & 0,21 & 0,21 & 0,25 & 0,31 \\
\hline Conteggio massimo & 20 & 13 & 30 & 40 & 80 \\
\hline \multicolumn{7}{|c|}{ Autunno/Autumn } \\
\hline Record & 101 & 98 & 146 & 202 & 240 \\
\hline Mediana conteggio & 2 & 1 & 1 & 2 & 1 \\
\hline SE & 0,23 & 0,11 & 0,13 & 0,22 & 0,17 \\
\hline Conteggio massimo & 15 & 6 & 8 & 25 & 24 \\
\hline
\end{tabular}

(Fig. 4A), con una maggiore concentrazione nelle risaie della provincia di Pavia, sia nel periodo primaverile che nel periodo autunnale: la specie è osservata in un ampio spettro di ambienti, comprendenti ripristini ambientali per avifauna acquatica, risaie, zone umide, sponde fluviali e lacustri, ex-cave naturalizzate, vasche di ex-zuccherifici, vasche di decantazione, foci fluviali, prati umidi in quota. Da rilevare come in periodo autunnale ci siano 16 record, 2 in provincia di Bergamo e 14 in provincia di Sondrio, prevalentemente in luglio ed agosto, localizzati ad oltre 2000 metri di quota, con valori massimi che superano i 2600 metri nei comuni di Bormio, Livigno, Valfurva (Azzalin D., Corti G., Del Togno R., Roverselli A.).

\section{Piro piro boschereccio}

Il numero di record del Piro piro boschereccio (Tab. 4) mostra un incremento costante tra il 2010 ed il 2013 (massimo di 297 in periodo primaverile e di 160 in pe- riodo autunnale): nel 2014 si registra un netto decremento delle osservazioni, molto più marcato nel periodo primaverile rispetto al periodo autunnale (Fig. 1B). A differenza del Piro piro culbianco, il Piro piro boschereccio mostra ben definiti periodi migratori (Fig. 2B), con inizio alla pentade 18 (27-31 marzo) ed una crescita costante con il massimo alla pentade 23 (21-25 aprile; massimo di 1740 individui), seguito da un veloce decremento coincidente con gli ultimi e scarsi contingenti migratori primaverili della pentade 28 (16-20 maggio). Il primo quartile rivela una variazione molto contenuta, compresa tra la giornata 101 (11 aprile) del 2010 e la giornata 95 (5 aprile) del 2014, con un valore mediano molto costante compreso in un range ristretto (giornate 112-114), ed anche il terzo quartile mostra una variazione molto contenuta, tra la giornata 125 e 129 (5-9 maggio; Fig. 3B). Le giornate delle prime osservazioni, sebbene variabili, non mostrano tendenze significative ad anticipare $(\mathrm{r}=-0,7238, \mathrm{t}=-1,82, \mathrm{P}=0,1663)$. Il movimento

Tab. 4 - Numero dei record, dimensione dei conteggi (mediana e valore massimo) di Piro piro boschereccio nel quinquennio 2010-2014 / Number of records and count results (median and maximum value) for the Wood Sandpiper in the period 2010-2014.

\begin{tabular}{|l|c|c|c|c|c|}
\hline \multicolumn{7}{|c|}{$\mathbf{2 0 1 0}$} & $\mathbf{2 0 1 1}$ & $\mathbf{2 0 1 2}$ & $\mathbf{2 0 1 3}$ & $\mathbf{2 0 1 4}$ \\
\hline \multicolumn{7}{|c|}{ Primavera/Spring } \\
\hline Record & 70 & 139 & 246 & 297 & 210 \\
\hline Mediana conteggio & 7 & 5 & 5 & 10 & 5 \\
\hline SE & 10,44 & 8,84 & 1,98 & 3,99 & 3,69 \\
\hline Conteggio massimo & 400 & 600 & 240 & 450 & 400 \\
\hline \multicolumn{7}{|c|}{ Autunno/Autumn } \\
\hline Record & 100 & 123 & 160 & 147 \\
\hline Mediana conteggio & 2 & 5 & 3 & 5 & 2 \\
\hline SE & 0,81 & 3,67 & 1,78 & 2,29 & 1,33 \\
\hline Conteggio massimo & 30 & 200 & 100 & 180 & 130 \\
\hline
\end{tabular}


Piro piro culbianco

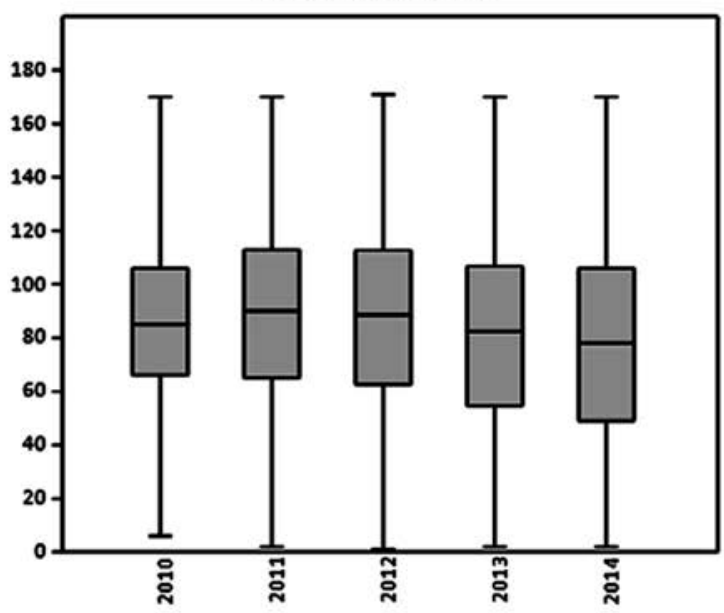

Pantana

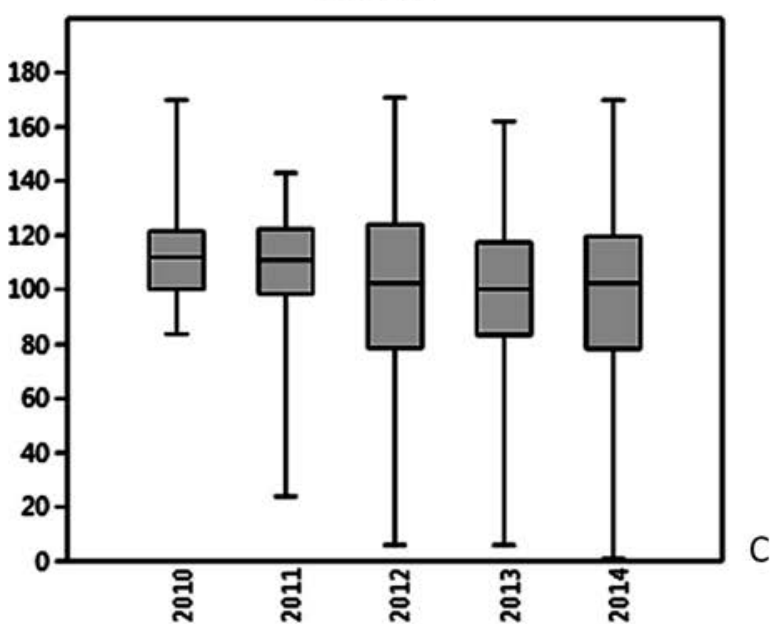

Pettegola

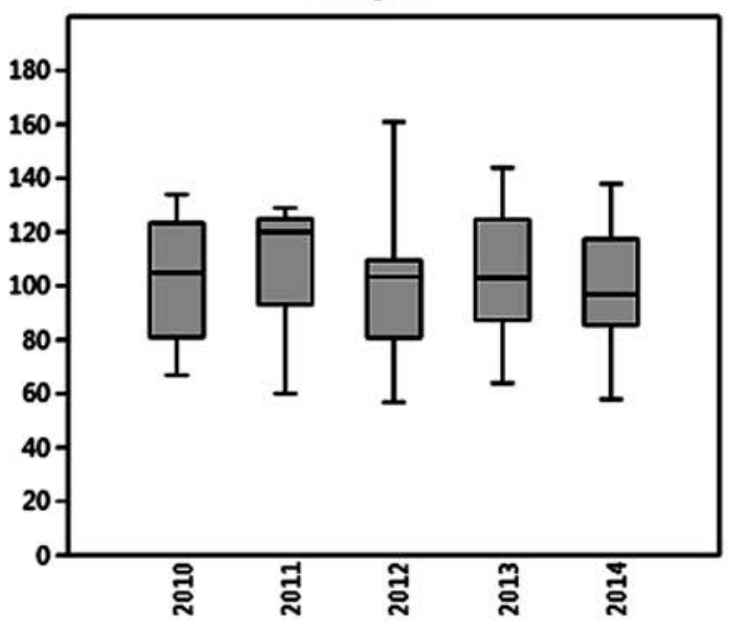

Piro piro boschereccio

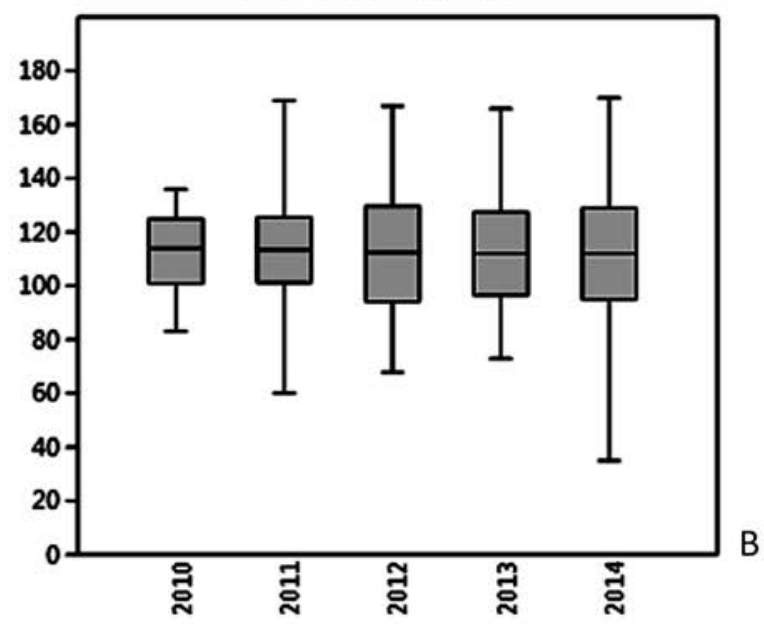

Totano moro

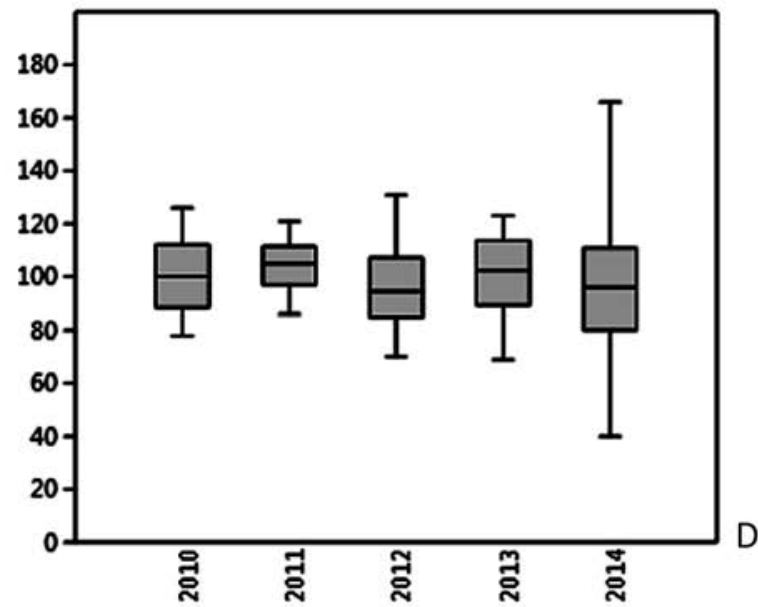

Albastrello

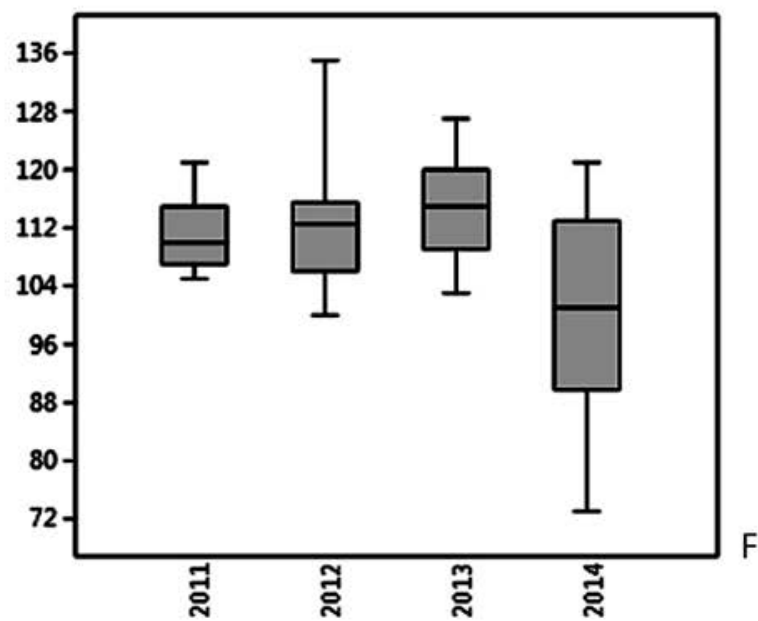

Fig. 4 - Grafici box-plot della variazione temporale della migrazione delle 6 specie: in ascissa l'anno, in ordinata il numero della giornata dell'anno. Il rettangolo indica il primo quartile, mediana e terzo quartile. I baffi indicano valore minimo e massimo della giornata dell'anno. / Box-plot graphs of migrating time variation for 6 species. The Y axis gives the day of year. Rectangles indicate the first quartile, median and third quartile, with bars indicating the minimum and maximum value for the day of year. 
autunnale, numericamente inferiore, è già evidente a partire dalla metà di giugno raggiungendo il massimo in corrispondenza della pentade 38 (5-9 luglio), per calare lentamente fino alla pentade 52 (13-17 settembre), con gli ultimi individui registrati nella pentade 7 (8-12 ottobre). Nessun record è stato registrato tra la pentade 58 (13-17 ottobre) e la fine d'anno per tutto il quinquennio. La specie è gregaria sia in periodo primaverile che in quello autunnale: sebbene in primavera i valori mediani dei conteggi siano compresi tra 5 e 10, ogni anno sono stati censiti gruppi numerosi, con un minimo di 240 nel 2012 (29 aprile, Risaie di C.na Torre Bianca in provincia di Pavia, Segagni D.) ed un massimo di 600 individui nel 2011, il 17 e 18 aprile presso le risaie di Cura Carpignano (PV; Segagni D.): le risaie della provincia di Pavia (Fig. 4B) risultano essere l'habitat elettivo della specie, e la quota media dei record oscilla tra gli 87 e i $125 \mathrm{~m}$ s.l.m. Molto scarsi sono i dati provenienti da elevate altitudini, con una osservazione 1' 8 agosto 2010 a Schilpario, a $1838 \mathrm{~m}$ (oss. Aguzzi S. ) ed un'altra il 21 agosto 2013, a Mazzo di Valtellina, a 1749 m (Corti G.). I conteggi nel periodo autunnale rivelano valori inferiori, sia nei massimi che nelle mediane: nel quinquennio da rilevare il conteggio di 200 individui, sempre in ambiente di risaia, il $1^{\circ}$ luglio (Segagni D.)

\section{Pantana}

Il numero dei record (Tab. 5) nel periodo primaverile mostra una crescita costante fino al 2013 (massimo di 271), seguito da un calo nel 2014; in periodo autunnale, dopo un biennio costante, la specie mostra un calo nel 2012, per riprendere nel biennio 2013/14 (Fig. 1C). La fenologia rivela un cospicuo flusso migratorio primaverile, mentre il passaggio autunnale non presenta particolari evidenze, essendo specie presente nel resto dell'anno con pochi individui (Fig. 2C). Si evidenzia un picco isolato ed anticipato alla pentade 15 (12-16 marzo) relativo all'anno 2011, relativo ad un osservazione di 100 individui nelle risaie di Confienza (PV, Ornaghi F.), mentre gli altri dati concorrono a delineare un incremento che inizia dalla pentade 18 (27-31 marzo) per arrivare al massimo compreso tra le pentadi 21 (11-15 aprile) e 24 (26-30 aprile), seguito da un decremento repentino in corrispondenza della prima decade di maggio (pentade 26). Gli individui presenti in periodo autunnale, in coincidenza delle pentadi 40/41 (dal 15 al 24 luglio) e $55 / 56$ (dal 28 settembre al 7 ottobre), superano di poco le 30 unità. La variazione del $1^{\circ}$ quartile, anticipando la data dei primi contingenti migratori, è evidente dal 2012 in avanti, passando dalla giornata 100 del 2010 alla 78 del 2014: la data della prima osservazione del 2010, alquanto ritardata, è imputabile a carenza di copertura, in considerazione dell'andamento sostanzialmente simile nel resto del quadriennio. I valori mediani mostrano una tendenza ad anticipare la parte centrale della migrazione (Fig. 3C), variando dalla giornata 112 del 2010 alla giornata 102 del 2014, e la tendenza risulta significativa $(\mathrm{r}=-0,957 ; \mathrm{t}=-5,734, \mathrm{P}=0,0105)$.

I conteggi massimi dei gruppi variano tra il massimo del biennio 2011/12 con 200 individui ed il minimo di 127 del 2014, ma la tendenza al decremento non è statisticamente significativa $(\mathrm{r}=0,014 ; \mathrm{t}=0,025, \mathrm{P}=0,9819)$. Le aree particolarmente vocate sono rappresentate sempre dalle risaie del Pavese (Fig. 4C): nel 2011 presso le camere di Cura Carpignano (il 17 aprile; Segagni D.) e nel 2012, tra il 2 ed il 9 aprile, presso Robbio (Bergamaschi L., Brigo M., Ornaghi F., Prada L.). L'unica segnalazione (Brigo M.) di Pantana in quota, si riferisce ad un individuo osservato il 27 agosto 2014, nel comune di Valdidentro (SO) a 1916 m s.l.m.

\section{Totano moro}

L'accumulo dei record per questa specie (Tab. 6) mostra andamenti differenti (Fig. 1D), evidenziando un incremento primaverile per il biennio 2012/13 (massimo di 146), seguito da un calo nel 2014, mentre per il periodo autunnale, i dati sono scarsi, con il numero maggiore (35) ottenuto per l'anno 2010, con una media annuale

Tab. 5 - Numero dei record, dimensione dei conteggi (mediana e valore massimo) di Pantana nel quinquennio 2010-2014. / Number of records and count results (median and maximum value) for the Greenshank in the period 2010-2014.

\begin{tabular}{|l|c|c|c|c|c|}
\hline \multicolumn{7}{|c|}{$\mathbf{2 0 1 0}$} & $\mathbf{2 0 1 1}$ & $\mathbf{2 0 1 2}$ & $\mathbf{2 0 1 3}$ & $\mathbf{2 0 1 4}$ \\
\hline Record & 41 & 97 & 218 & 271 & 201 \\
\hline Mediana conteggio & 3 & 5 & 2 & 4 & 3 \\
\hline SE & 2,69 & 3,71 & 1,96 & 1,4 & 1,37 \\
\hline Conteggio massimo & 100 & 200 & 200 & 150 & 127 \\
\hline \multicolumn{7}{|c|}{ Autunno/Autumn } \\
\hline Record & 55 & 66 & 33 & 87 & 133 \\
\hline Mediana conteggio & 2 & 2 & 1 & 2 & 1 \\
\hline SE & 0,42 & 0,24 & 0,25 & 0,46 & 0,35 \\
\hline Conteggio massimo & 15 & 10 & 6 & 20 & 19 \\
\hline
\end{tabular}


Tab. 6 - Numero dei record, dimensione dei conteggi (mediana e valore massimo) di Totano moro nel quinquennio 2010-2014. / Number of records and count results (median and maximum value) for the Spotted Redshank in the period 2010-2014.

\begin{tabular}{|l|c|c|c|c|c|}
\hline \multicolumn{7}{|c|}{$\mathbf{2 0 1 0}$} & $\mathbf{2 0 1 1}$ & $\mathbf{2 0 1 2}$ & $\mathbf{2 0 1 3}$ & $\mathbf{2 0 1 4}$ \\
\hline \multicolumn{7}{|c|}{ Primavera/Spring } \\
\hline Record & 32 & 35 & 88 & 146 & 103 \\
\hline Mediana conteggio & 2,5 & 4 & 1,5 & 5 & 2 \\
\hline SE & 3,54 & 6,23 & 3,61 & 2,43 & 2,94 \\
\hline Conteggio massimo & 100 & 200 & 200 & 250 & 239 \\
\hline \multicolumn{7}{|c|}{ Autunno/Autumn } \\
\hline Record & 35 & 10 & 28 & 26 & 16 \\
\hline Mediana conteggio & 2 & 1 & 2 & 1 & 1 \\
\hline SE & 0,94 & 1,5 & 0,98 & 0,74 & 0,35 \\
\hline Conteggio massimo & 23 & 16 & 28 & 20 & 6 \\
\hline
\end{tabular}

di $23,3(\mathrm{DS}=6,43)$ tra il 2012 ed il 2014. Il movimento del Totano moro si concentra quasi esclusivamente nel periodo primaverile (Fig. 5A), con inizio alla pentade 17 (22-26 marzo) ed un doppio picco dovuto allo sfasamento dei massimi per il biennio 2011/2012 (pentadi 18 e 19, dal 27 marzo al 5 aprile) con quello del biennio 2013/2014 (pentadi 21 e 22, dall' 11 al 20 aprile); tra la pentade 23 e 25 inizia la fase discendente che termina bruscamente dopo la pentade 26 (6-10 maggio). Seppure con piccoli numeri di individui il movimento autunnale è rilevabile tra la pentade 48 (2-28 agosto) e la pentade 57 (8-12 ottobre), per terminare quasi completamente verso la fine dell'anno. Tra il 2010 ed il 2014 è rilevabile un leggero incremento dei record compresi tra la metà di ottobre e la fine d'anno, con alcuni individui osservati anche nel mese di dicembre nel biennio 2013/2014, nelle Cave di San Gaudenzio (comune di Cervesina, PV; Assandri G., Bernini F., Tiso E.) e, solo per il 2014, a Cassinazza di Baselica (comune di Giussago, PV; Janni O.); la tendenza non è significativa (Kruskal-Wallis, $\mathrm{H}=5,54$, $\mathrm{P}=0,2362)$. La data mediana della migrazione primaverile risulta stabile (Fig. 3D), variando dalla giornata 100 del 2010 alla 96 del 2014 (Kruskal-Wallis $\mathrm{H}=6,42$, $\mathrm{P}=0,1699$ ) ed anche il $1^{\circ}$ quartile segue tale andamento, passando dalla giornata 88 alla 80 , sebbene sia evidenziabile un anticipo delle date delle prime osservazioni, passando dalla giornata 78 (19 marzo) del 2010 alla giornata 40 ( 9 febbraio) del 2014, ma la tendenza non risulta significativa $(\mathrm{r}=-0,845, \mathrm{P}=0,0715)$. Il Totano moro transita in gruppi medio/piccoli come evidenzia il valore della mediana nel quinquennio, con un massimo di 250 il 17 aprile 2013, nelle risaie di Confienza (PV, Brigo M.). La parte nord-occidentale della provincia di Pavia, con le risaie dei comuni di Confienza e Robbio (Fig. 4D), è il settore che nel quinquennio raccoglie $\mathrm{i}$ gruppi di dimensioni maggiori e tutti i valori massimi sono ascrivibili a questi due comuni. Al di fuori dell'area risicola da evidenziare il gruppo di 32 individui, presente nelle vasche dell'ex-zuccherificio di Casei Gerola (PV), tra il 21 ed il 24 aprile 2012 (pentade 23; Binari U., Tiso E.), ed il gruppo di 22, il 21 aprile 2014, nella Riserva Naturale delle Torbiere del Sebino, nel comune di Provaglio d'Iseo (BS, de Pascali F.). I dati della specie sono tutti compresi entro i 200 metri di quota, con un massimo registrato di $204 \mathrm{~m}$ s.l.m. per il comune di Sorico, il 21 aprile 2013 (1 individuo, Viganò E.)

\section{Pettegola}

Il numero dei record (Tab. 7) per questa specie mostra un incremento nel periodo primaverile nel biennio 2012/13, per calare successivamente nel 2014; per il periodo autunnale i dati sono molto scarsi per tutto il quinquennio (Tab. 5 e Fig. 1D). La Pettegola mostra un range temporale di migrazione primaverile abbastanza ampio, con movimenti che iniziano alla pentade 12 (25 febbraio-1 marzo), con un incremento in corrispondenza della pentade 17 (Fig. 5B) ed un picco alla pentade 19 (1-5 aprile): l'andamento mostra un rallentamento nel passaggio degli individui in corrispondenza delle pentadi 22 e 23 (dal 16 al 25 aprile), fattore comune a tutto il quinquennio, mentre il picco inferiore corrispondente alla pentadi 24 (26-30 aprile) e 25 (1-5 maggio), corrisponde all'anno 2014. Il passaggio autunnale è molto meno evidente ed è concentrato tra la pentade 38 (5-9 luglio) e la 52 (13-17 settembre); dalla pentade 61 (28 ottobre-1 novembre) alla fine dell'anno non esistono osservazioni. Nel quinquennio (Fig. 3E) il primo quartile mostra una fluttuazione tra la giornata 81 (22 marzo) e la 93 ( 3 aprile) mentre i valori mediani variano tra la giornata 120 (30 aprile) del $2011 \mathrm{e}$ la 97 (7 aprile) del 2014, con una tendenza non significativa (Kruskal-Wallis $\mathrm{H}=5,77, \mathrm{P}=0,217$ ); il terzo quartile mostra variazioni tra la giornata 109 (19 aprile) del 2012 e la 125 del 2011 (5 maggio). La prima osservazione varia tra la giornata 57 del 2012 (29 marzo) e la 67 del 2010 (7 marzo). La specie è presente solitamente con individui isolati o gruppi molto piccoli, generalmente inferiori a 5 e raramente superiori a 10 , come confermano $i$ valori mediani dei conteggi primaverili (Tab. 5); il massimo è stato di 30 individui ( 3 aprile 2013, Casale F., Cassolno- 

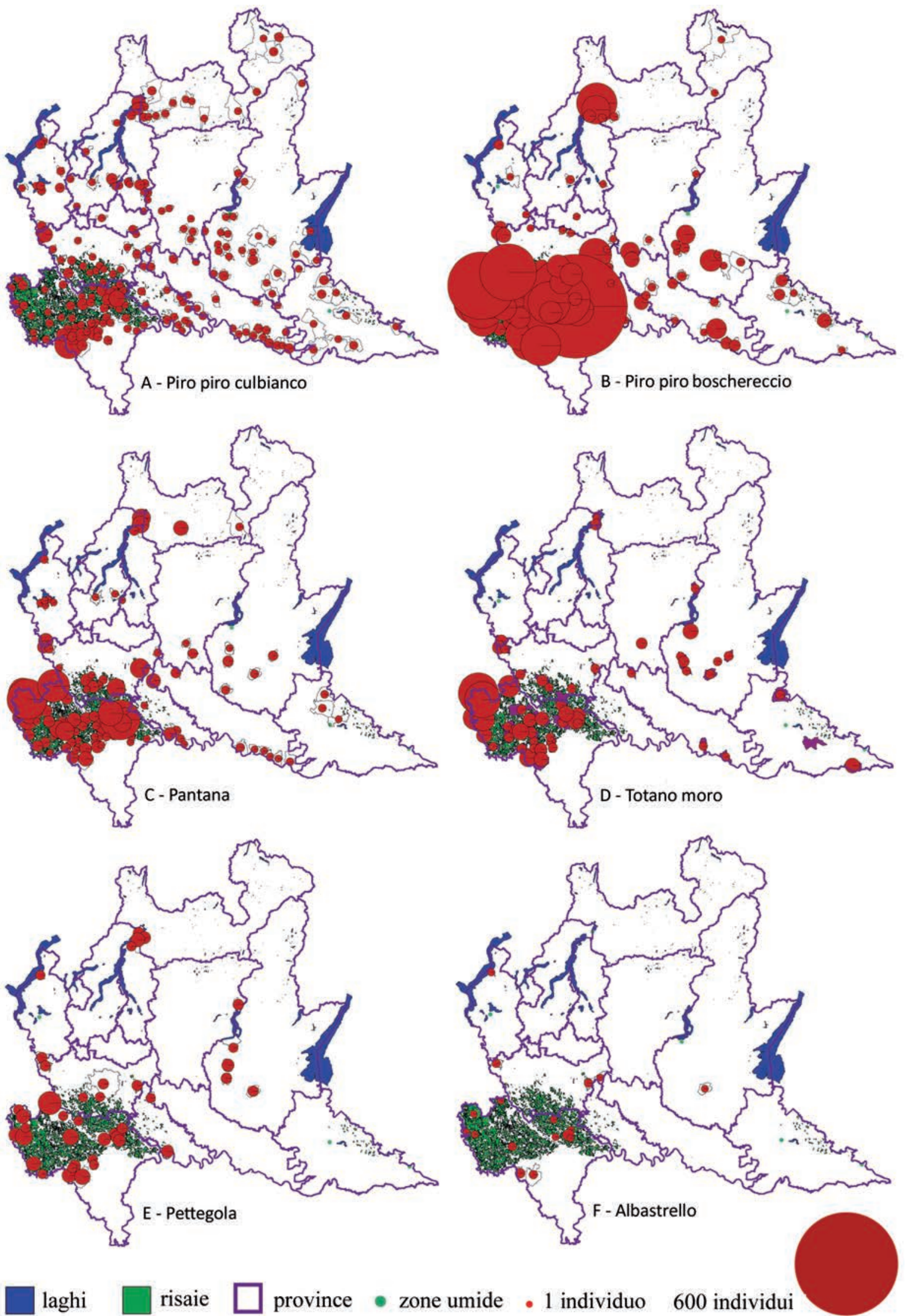

laghi risaie province $\bullet$ zone umide $\bullet 1$ individuo

individu

Fig. 5 - Numero massimo di individui osservati nel quinquennio 2010-2014 durante la migrazione primaverile: il diametro dei cerchi rossi corrisponde al numero massimo di individui per comune, variando in un range compreso tra 1 (valore minimo) e 600 (valore massimo). / Maximum number of individuals observed during spring migration over the five-year period (2010-2014). Red circle diameter corresponds to the maximum number of individuals per municipality, ranging from 1 (minimum) to 600 (maximum). 
Tab. 7 - Numero dei record, dimensione dei conteggi (mediana e valore massimo) di Pettegola nel quinquennio 2010-2014. / Number of records and count results (median and maximum value) for the Redshank in the period 2010-2014.

\begin{tabular}{|l|c|c|c|c|c|}
\hline & 2010 & 2011 & 2012 & 2013 & 2014 \\
\hline \multicolumn{5}{|c|}{ Primavera/Spring } \\
\hline Record & 24 & 21 & 41 & 85 & 55 \\
\hline Mediana conteggio & 1 & 2 & 1 & 1 & 1 \\
\hline SE & 0,58 & 0,49 & 0,21 & 0,39 & 0,52 \\
\hline Conteggio massimo & 11 & 10 & 7 & 30 & 17 \\
\hline \multicolumn{7}{|c|}{ Autunno/Autumn } \\
\hline Record & 11 & 6 & 9 & 12 & 6 \\
\hline Mediana conteggio & 1 & 1,5 & 1 & 1 & 1 \\
\hline SE & 0,28 & 0,63 & 0,11 & 0,56 & 0,17 \\
\hline Conteggio massimo & 4 & 5 & 2 & 6 & 2 \\
\hline
\end{tabular}

vo, PV). In autunno il numero di individui presenti cala ulteriormente, con un massimo di 6, osservati a Lonate Pozzolo (VA, Tomasi M.) il 9 luglio. La distribuzione geografica interessa essenzialmente le aree risicole, fluviali ed i ripristini ambientali della provincia di Pavia e di Milano (Fig. 4E), con alcuni individui in transito nelle zone umide del Pian di Spagna/foce Adda (CO), presso la Riserva Naturale delle Torbiere del Sebino, la foce del fiume Tresa e Lonate Pozzolo (VA). Al pari del Totano moro le osservazioni della Pettegole sono comprese entro i 200 metri di quota, con un unico dato relativo al comune di Dubino (203 m s.l.m., SO; 7 aprile 2013, Corbellini G.).

\section{Albastrello}

L'incremento primaverile dei record della specie mostra una crescita significativa nel quinquennio $(r=0,946$, $\mathrm{P}=0,015$ ), a cui si contrappone un decremento nel periodo autunnale, particolarmente evidente nel biennio 2013/14.
Il numero degli individui è sempre comunque molto basso (Tab. 8 e Fig. 1E) con i valori delle mediane dei conteggi pari sempre ad 1 ad esclusione della primavera 2010, con un massimo di 13 individui (12 settembre 2012, Cave di San Gaudenzio 2, PV, Binari U.). Lo scarso passaggio primaverile è compreso prevalentemente tra la pentade 19 (1-5 aprile) e la pentade 27 (11-15 maggio), senza picchi evidenti (Fig. 5C), mentre il periodo autunnale raccoglie saltuarie osservazioni comprese tra la pentade 36 (25-29 giugno) e la 52 (13-17 settembre), senza alcun altro dato fino alla fine dell'anno per tutto il quinquennio. Lo scarso numero di record rivela un'ampia variabilità, evidenziata nel box-plot di Fig. 3F. Come mostra la carta della distribuzione geografica dei record (Fig. 4F) l'Albastrello frequenta esclusivamente aree planiziali entro i $100 \mathrm{~m}$ s.l.m., situate nelle province di Pavia, Milano e Brescia: l'unico dato del quinquennio raccolto in provincia di Varese si riferisce ad una quota di $194 \mathrm{~m}$ (foce del fiume Tresa, 12 aprile 2013, Carabella M.).

Tab. 8 - Numero dei record, dimensione dei conteggi (mediana e valore massimo) di Albastrello nel quinquennio 2010-2014 / Number of records and count results (median and maximum value) for the Marsh Sandpiper in the period 2010-2014.

\begin{tabular}{|l|c|c|c|c|c|}
\hline \multicolumn{7}{|c|}{$\mathbf{2 0 1 0}$} & $\mathbf{2 0 1 1}$ & $\mathbf{2 0 1 2}$ & $\mathbf{2 0 1 3}$ & $\mathbf{2 0 1 4}$ \\
\hline Record & 1 & 9 & 8 & 13 & 16 \\
\hline Mediana conteggio & 3 & 1 & 1 & 1 & 1 \\
\hline SE & 0 & 0,46 & 0,27 & 0,1 & 0,12 \\
\hline Conteggio massimo & 3 & 5 & 3 & 2 & 2 \\
\hline \multicolumn{7}{|c|}{ Autunno/Autumn } \\
\hline Record & 7 & 6 & 7 & 1 & 0 \\
\hline Mediana conteggio & 1 & 1 & 1 & 1 & - \\
\hline SE & 1 & 0 & 1,7 & 0 & - \\
\hline Conteggio massimo & 8 & 1 & 13 & 1 & - \\
\hline
\end{tabular}




\section{DISCUSSIONE}

Il flusso migratorio dei limicoli che interessa la regione Lombardia è ascrivibile sia alla flyway del Mar Nero/Mediterraneo che a quella dell'Atlantico Orientale (Boere \& Stroud, 2006): per il genere Tringa le ricatture di individui esteri ripresi in Italia (Spina \& Volponi, 2008) mostrano un'origine prevalente dall'Europa centro-settentrionale e parzialmente dal settore orientale, relativo a Finlandia, Polonia e paesi baltici. Nell'ambito delle flyways continentali, la distribuzione geografica dei dati presentati in questo lavoro rivela l'importanza degli ambienti di origine artificiale, risaie e ripristini ambientali, della pianura padana lombarda, relativamente povera delle adatte zone umide naturali e degli habitat deltizi: questo fattore può condizionare le dimensioni dei contingenti migratori e svernanti per Totano moro e Pettegola, favorendo rotte più spostate ad est, verso le vaste zone umide salmastre, le saline ed i litorali marini dell'alto Adriatico, lungo l'arco compreso tra Emilia Romagna e Friuli Venezia Giulia. Rispetto ad altre aree continentali europee, rappresentate dalle lagune costiere di Spagna e Francia a Ovest, di Grecia e Montenegro ad Est, e dal vastissimo sistema costiero intertidale del Mare del Nord, esteso dall'Olanda alla Danimarca, solo un sito italiano risulta essere un Key-Site che soddisfa il criterio dell' $1 \%$ per le dimensioni della popolazione presente: la Laguna di Grado e Marano per il Totano moro (Delany et al., 2009); da rilevare però come tale valore si riferisca ad un conteggio effettuato nel 1993. Nonostante questo fattore di scala e latitudine, il distretto risicolo lombardo, ecologicamente compreso nella più vasta area lombardo-piemontese, risulta una fondamentale area di stopover, in particolare nel corso della migrazione pre-nuziale.

Nell'anno 2014 la superficie risicola lombarda ammontava a 90.780 ettari (www.enterisi.it), distribuiti prevalentemente in provincia di Pavia $(84.9 \%)$, secondariamente in quella di Milano (13.3\%), con una scarsa frazione in quella di Lodi (1.7\%), mentre per il Piemonte il totale complessivo raggiungeva i 112.510 ettari, concentrati in maggioranza in provincia di Vercelli; in totale la superficie delle due regioni totalizzava 203.290 ettari. É noto come le risaie allagate costituiscano un valido surrogato delle zone umide per molti uccelli acquatici (Fasola et al., 1996; Fasola \& Ruiz, 1996; Lawler, 2001; Longoni, 2010; Toral \& Figuerola, 2010; Longoni et al., 2013), sia durante la migrazione che nel corso della nidificazione: le pratiche agricole possono però compromettere la funzionalità dell'habitat, attraverso il livellamento laser delle camere, l'uso massiccio di pesticidi ed erbicidi, la coltivazione in asciutta, la successione delle operazioni (Lawler, 2001; Lourenço \& Piersma, 2009; Ibañez et al., 2010; Cardarelli et al., 2013). La coltivazione in asciutta, con le ricadute dirette sulle potenzialità trofiche del sistema, si configura come una tendenza crescente: nel distretto risicolo lombardo piemontese è passata dal $16 \%$ del totale coltivato nel 2004 al 33\% del 2014 (Ranghetti et al., 2015). Una significativa proporzione delle osservazioni provengono dai ripristini ambientali esistenti nella parte occidentale della regione: dal Parco Sovracomunale le Folaghe di Casei Gerola con le adiacenti vasche dell'ex-zuccherificio, alle Cave di San Gaudenzio in co- mune di Cervesina, all'Azienda Agroambientale della Cassinazza di Baselica in comune di Giussago, siti tutti localizzati in provincia di Pavia. Per l'area di Milano vanno citate le vasche di laminazione e spagliamento del torrente Arno, comunemente denominate Vasche dell'Arnetta. La fenologia della migrazione primaverile e le dimensioni dei gruppi per Totano moro, Pettegola, Albastrello e Piro piro culbianco, risultano molto simili a quanto riportato per la regione Piemonte: nel periodo 2010-2012 i conteggi massimi per il distretto risicolo piemontese indicavano un valore massimo di 250 individui per il Totano moro (23 aprile 2011, provincia di Novara) e 30 individui per la Pettegola il 6 maggio dello stesso anno, nella provincia di Cuneo (Alessandria et al., 2012). Per il Totano moro era stato rilevato un picco nel 2008, nelle risaie del Vercellese-Novarese, nell'ultima decade di aprile (Toffoli, 2008): per questa specie alcuni dati raccolti in un monitoraggio della migrazione primaverile nel marzo-maggio del 1990, confermano un rapido passaggio dei contingenti migratori in Italia settentrionale, con valori di picco centrati sulla pentade 23 (21-25 aprile), sia nelle risaie del Vercellese che nelle saline di Cervia (Serra et al., 1992). Le presenze più consistenti in Italia sono localizzate nelle zone umide costiere del Tirreno e dell'Adriatico, in quest'ultimo settore per quanto riguarda lo svernamento (Brichetti \& Fracasso, 2004; Tinarelli et al., 2010); sul versante opposto dell'Adriatico, presso le saline costiere di Ulcinj nel Montenegro, si concentra anche un importante flusso della migrazione post-riproduttiva, con oltre 2200 individui rilevati nel 2006 (Schneider-Jacoby \& Saveolic, 2007). La maggior parte delle ricatture di individui inanellati all'estero, effettuate in periodo autunnale e dovute ad abbattimento diretto, proviene dall'Europa centro settentrionale, in particolare da Svezia e Finlandia (Spina \& Volponi, 2008). Sebbene la specie non presenti problemi di conservazione nella porzione europea del Paleartico Occidentale, inclusi i paesi dell'ex-federazione sovietica, recentemente il Totano moro ha visto elevarsi il livello di minaccia, passando da LC (Least Concern) a NT (Near Threatened; BirdLife International, 2015) in relazione alla diminuzione della popolazione continentale. A conferma di ciò anche il trend della popolazione in transito nei due periodi migratori nel settore tedesco del Wadden Sea, mostra un importante declino a partire dal 1990 (Hötker et al., 2010). Analogo ed ulteriore incremento del grado di minaccia, alla medesima scala spaziale, lo subiscono sia la Pettegola che passa da LC a VU (Vulnerable), sia l'Albastrello che, da LC, approda a EN (Endangered). La Pettegola ha un'ampia distribuzione europea, ma per diverse popolazioni Wetland International indica una tendenza al decremento sia nella parte centro-settentrionale che in quella orientale del continente (www.wow.wetlands.org). In Italia è anche specie nidificante nelle lagune costiere dell'alto Adriatico, con una cospicua popolazione concentrata nella laguna di Venezia: nel periodo 2001-2006 era stata censita una media di 1593 coppie (Scarton et al., 2008). La provenienza delle ricatture (principalmente abbattimenti) effettuate in Italia è ascrivibile all'Europa centrosettentrionale, con una maggioranza di individui di origine danese (Spina \& Volponi, 2008). Anche in Piemonte 
l'Albastrello, nel periodo 2010-2012, è riportato con uno scarso numero di individui, con un massimo di 10 (3 aprile 2011, provincia di Novara: Alessandria et al., 2012); in Italia è più frequente durante le migrazioni nelle lagune della costa adriatica, in cui sono riportate concentrazioni di alcune centinaia di individui (Brichetti \& Fracasso 2004): nell'ambito della flyway della specie l'unico keysite che soddisfa il criterio dell' $1 \%$ nell'Europa continentale si identifica nell'area della Camargue (www.wow. wetlands.org). Nel continente europeo il Piro piro culbianco frequenta prevalentemente le acque interne ed è un migratore precoce come confermano i dati di questo lavoro, sia nella migrazione primaverile che in quella autunnale, con individui in movimento già a giugno (Delany et al., 2009). Anche in Piemonte l'ordine dei grandezza dei gruppi osservati nel periodo 1995-2000 (Vaschetti et al., 2001) e nel 2010 (Alessandria et al., 2011) è paragonabile a quanto emerge dai dati lombardi per il quinquennio in esame con, rispettivamente, un conteggio massimo di 38 individui nel luglio ed agosto del 1997, ed ancora 40 e 29 individui il 10 e 29 luglio del 2010. La continua presenza della specie in periodo autunnale, evidente nel grafico di Fig. 2A, è emersa anche da uno studio condotto nella Valle del Reuss, nel Cantone Zurigo in Svizzera settentrionale, nel periodo 1971-1993: con inizio nella seconda metà di giugno, il passaggio degli individui raggiunge un picco alla fine di luglio, in corrispondenza della pentade 43, decrescendo lentamente fino a novembre (Schelbert et al., 1995). Le principali aree di svernamento della specie in Italia, sono costituite dalle lagune costiere dell'alto Adriatico e dalla pianura padana centro-occidentale (Brichetti \& Fracasso, 2004). Per il Piro piro boschereccio, rispetto ai valori massimi registrati nel quinquennio in Lombardia, i dati per la regione Piemonte mostrano valori dei conteggi giornalieri ancor più elevati: 1310 individui il 24 aprile 2010 (Alessandria et al., 2011) e, nello stesso giorno del 2011, 2830 individui nelle risaie del vercellese (Alessandria et al., 2012), in corrispondenza della pentade 23 , in accordo con quanto fornito dai dati lombardi. Questi valori massimi piemontesi sono da 3 ad 8 volte superiori a quanto riportato per le risaie di Roncaro (massimo di 390 individui il 21 aprile 2010) e Cura Carpignano (massimo di 350 il 22 aprile 2011), situate tra i 60 e i $90 \mathrm{~km}$ da quelle Vercellesi. Questa ampia differenza potrebbe essere spiegata sia con il rapido spostamento dei contingenti migratori tra un settore e l'altro, sia con condizioni trofiche particolarmente favorevoli nelle risaie vercellesi ma, in mancanza di un marcaggio e ricattura degli individui, è difficilmente dimostrabile. Sempre nella Valle del Reuss, nel periodo 1971-1993, i valori di picco sono stati registrati tra le pentadi 24-26 (Schelbert et al., 1995), in concordanza con uno spostamento progressivo verso nord. Valori mediani della migrazione primaverile indicano la data dell' 8 maggio per il periodo 1981-2000, in un impianto di trattamento delle acque a Munster, nella Renania Settentrionale-Vestaflia, nella Germania occidentale (Anthes et al., 2002). In autunno il movimento di ritorno in Lombardia ha già inizio alla fine di giugno, in corrispondenza delle pentadi 35 e 36: 1'1 luglio 2011 (pentade 37), a S. Alessio con Vialone (PV) erano già in transito 200 individui (Se- gagni D.), in anticipo rispetto a quanto citato per la Pianura Padana centrale, in provincia di Modena, con i primi individui osservati tra il 10 ed il 14 Luglio (Brichetti \& Fracasso, 2004). A livello europeo il movimento autunnale avviene prevalentemente in agosto-metà settembre, ma anche in Camargue gli adulti arrivano già a luglio, un mese prima dei giovani, per accumulare riserve di grasso prima di intraprendere la traversata del Mediterraneo e del Sahara (Delany et al., 2009). La maggioranza degli individui inanellati ripresi in Italia in periodo autunnale proviene dall'Europa centro-settentrionale, con una netta prevalenza dai Paesi del Mar Baltico, in particolare dalla Svezia (Spina \& Volponi, 2008). Anche per la Pantana vale quanto indicato per il Piro piro boschereccio durante la migrazione primaverile: i numeri massimi rilevati in Piemonte sono alquanto superiori rispetto alla Lombardia, con valori che arrivano a 1350 individui il 17 aprile 2010, a 1340 il 24 aprile 2011, sempre in provincia di Vercelli (Alessandria et al., 2011, 2012). Il movimento primaverile della specie in Europa è concentrato in aprile (Delany et al., 2009), ma i dati lombardi indicano un cospicuo incremento già alla pentade 18 (27-31 marzo), con i valori massimi comunque compresi nel mese di aprile, in concordanza con quanto riportato per le lagune costiere del Tirreno e dell'Adriatico (Brichetti \& Fracasso, 2004). Il transito autunnale non presenta invece particolare intensità, legato probabilmente più alle coste atlantiche europee del Mare del Nord, come evidenziano la maggioranza dei key-sites ubicati lungo le coste del Wadden Sea, dall'Olanda alla Danimarca (Delany et al., 2009). Le scarse ricatture $(n=29)$ di individui inanellati all'estero indicano una provenienza centro-europea (Germania, Repubblica Ceca, Polonia), con un influsso anche dalla Finlandia (Spina \& Volponi, 2008).

L'analisi dei dati effettuata in questo lavoro dimostra come la citizen science possa contribuire sostanzialmente all'incremento delle conoscenze faunistiche di base: solo pochi anni fa un risultato simile sarebbe stato impensabile ed il progressivo aumento del numero degli iscritti consentirà una migliore ed ancora più vasta copertura del territorio. La discrepanza nei conteggi di alcune specie, rilevata rispetto alle aree risicole piemontesi per il biennio 2010/11, potrebbe essere dovuta anche alla scarsa copertura iniziale dovuta al basso numero di osservatori, il cui numero è andato costantemente aumentando nel corso del quinquennio, passando dai 58 del 2010 ai 165 del 2014. La mancanza di un piano standardizzato di rilevamento può senz'altro aver influito sui risultanti ottenuti: a tal fine sarebbe utile poter disporre di conteggi contemporanei, ripetuti negli anni, effettuati nelle principali aree interessate dalla migrazione.

\section{Ringraziamenti}

Sono molto grato al gruppo di conduzione di Ornitho. it per aver reso disponibili i dati del genere Tringa, in particolare a Roberto Lardelli per la collaborazione dimostrata. Ringrazio Roberto Aletti per il costruttivo scambio di idee relativo all'identificazione geografica della località, Mauro Fasola per le indicazioni bibliografiche, Marco Pavia per le informazioni fornite. Ringrazio i referees e 
Giuseppe Bogliani per le utili osservazioni atte a migliorare la prima stesura del lavoro. Il mio sincero apprezzamento a tutti gli ornitologi e osservatori che costituiscono la forza reale della citizen science: Aguzzi Stefano, Aldi Davide, Aletti Roberto, Andena Luigi, Assandri Giacomo, Azzalin Dario, Balbo Simone, Bandera Fabrizio, Bassi Enrico, Bassi Silvio, Battisti Andrea, Bazzi Gaia, Bazzi Lionello, Belardi Mauro, Bellintani Stefano, Benazzo Massimo, Benetti Claudio, Bergamaschi Luca, Bernadara Enos, Bernasconi Domenico, Bernini Franco, Bertini Paolo, Bertoli Roberto, Binari Umberto, Bogliani Giuseppe, Boldrini Alex, Bonanno Laura, Bonazzi Paolo, Bondioli Simona, Bongio Mattia, Bonifacino Marco, Bonomelli Luca, Bontempi Marco, Bonvicini Piero, Bozzetti Antonio, Brambilla Mattia, Brambilla Olivia, Brembilla Roberto, Bressan Paola, Brezzi Fabio, Brichetti Pierandrea, Brignoli Gianpaolo, Brigo M. Luigi, Burini Maurizio, Canziani Mauro, Capelli Federico, Capelli Stefania, Carabella Monica, Carpi M. Grazia, Casale Fabio, Cavallini Ernesto, Castelli Gianluigi, Cavazioni Giancarlo, Cavenaghi Alberto, Cennamo Raffaele, Chemollo Marco, Chesini Luigi, Chiari Carlo, Chiatante Gianpasquale, Cima Guido, Cirolo Alfonso, Clerici Walter, Colaone Silvio, Conca Giovanni, Conti C. Alberto, Corbellino Gabriele, Cordioli Gianluca, Cornalba Maurizio, Corno Giuditta, Cortemiglia Marco, Corti Gianpaolo, Cosco Mariantonella, Costagli Massimo, Costanzo Alessandra, Crivellari Marco, Crocetti Silvia, Damiano Corrado, Danielli Simona, Delle Monache Antonio, Delle Vedove Giangaetano, de Pascalis Federico, Del Togno Riccardo, Dell'Acqua Cesare, Dellera Alvaro, Demartini Luigi, Di Liddo Giorgio, Di Pietra Francesco, Di Rienzo Alfonso, Ellwanger Götz, Faccaro Stefano, Facchetti Davide, Faifer Paolo, Falaschi Mattia, Ferlini Flavio, Ferlini Carlo Maria, Foglini Claudio, Fontana Simone, Forlani Emanuele, Franzoi Alessandro, Gagliardi Alessandra, Galimberti Andrea, Galuzzi Federico, Ganassi Oliviero, Gandini Mario, Gargioni Arturo, Gariboldi Armando, Gatti Francesco, Giannella Carlo, Giussani Enrico, Giussani Luca, Ghisolfi Marco, Gobbini Marco, Grattini Nunzio, Guenzani Gualtiero, Hörmann Bettina e Peter, Ilahiane Luca, Izzo Cristiano, Janni Ottavio, Ken Montadon, Laddaga Lorenzo, Lancini Gianfranco, Leoni Giorgio, Lerco Roberto, Levert Regis, Liebel Heiko, Longo Marco, Longoni Violetta, Lovisetto Fabio, Lupi Roberto, Lucarelli Stefano, Luoni Federica, Madella Alessandro, Maggioni Annalisa, Manfredini Edoardo, Marocchi G.Andrea Roberto, Mantovani Cristiano e Sergio, Martinoli Alessio, Massalongo Diego, Mattinelli Alberto, Mazzi Giorgio, Mazzoleni Alessandro, Mazzotti Sergio, Micheli Alessandro, Monfrini Mario e Stefano, Moranti Michele, Mozzetti Ettore, Mezzomo Luca, Motta Gabriella, Natale Giovanni, Nava Gaetano, Negra Osvaldo, Nicoli Andrea, Nicolodi Stefano, Nigro Luca, Orlandi Mauro, Ornaghi Francesco, Pagliari Gabriele, Papale Gabriele, Parisi Andrea, Passarotto Arianna, Pellegrini Francesco, Perin Vincenzo, Pietrobon Aldo, Pinoli Guido, Pianezza Federico, Pietrobon Aldo, Piretta Lorenza, Pirotta Giuliana, Pistono Carlo, Porta Dario, Pozzi Gianni, Prada Lorenzo, Prestileo Andrea, Provini Ivan, Quaranta Dario, Ravizza Luca, Razzetti Edoardo, Redaelli Giuseppe, Reggiani Alessandro, Regusoni Fabio, Riboni Bassano, Ricci
Marco, Rigamonti Ettore, Ronchi Matteo, Ronconi Daniele, Rota Roberto, Rovelli Cesare, Roverselli Andrea, Rubolini Diego, Rudolf Kummel, Ruggieri Luciano, Sala Alfio, Saporetti Fabio, Saporiti Vittorio, Sassi Walter, Sbravati Cristiano, Scalzotto Fabio, Schenk Norbert, Segagni Domenico, Sesenna Maurizio, Sighele Maurizio, Siliprandi Marco, Simonini Giacomo, Sozzi Marco, Speranza Giuseppe, Stella Lucia, Strada Amleto, Sutti Paolo, Tarozzi Andrea, Taverna Giuseppe, Tellini Florenzano Guido, Tiso Eugenio, Tomasi Mirko, Trotti Paolo, Tucci Riccardo, Turconi Giuliana, Usubelli Fabrizio, Vailati Morena, Valfiorito Rudy, Vidolini Andrea, Viganò Enrico, Viganò Michele, Vigo Enzio, Vitulano Severino, Zimbelli Alfredo, Zanini Corrado.

\section{BIBLIOGRAFIA}

Alessandria G., Della Toffola M. \& Fasano S., 2011 Resoconto ornitologico per la regione Piemonte-Valle d'Aosta. Anno 2010. Rivista Piemontese di Storia Naturale, 32: 297-351.

Alessandria G., Caprio E., Della Toffola M., Fasano S.G. \& Pavia M., 2012 - Resoconto ornitologico per la regione Piemonte-Valle d'Aosta. Anno 2011. Rivista Piemontese di Storia Naturale, 33: 337-395.

Anthes N., Harry I., Mantel K., Müller A., Schielzeth H. \& Wahl J., 2002 - Notes on migration dynamics of the Wood Sandpiper (Tringa glareola) at the sewage farm of Münster (NW Germany). Ring, 24: 41-56.

Baietto M., 2005 - Gli Uccelli Acquatici del Parco Agricolo Sud Milano. Parco Agricolo Sud Milano, Provincia di Milano, Milano.

Baietto M. \& Bottoni L., 2005 - Censimento degli uccelli acquatici migratori nelle risaie del Parco Agricolo Sud Milano. Avocetta, 29: 44.

Barbieri F., 1988 - Uccelli acquatici svernanti in Lombardia. Atti del IV Convegno Italiano di Ornitologia. Il Naturalista Siciliano, 12.

Berthold P., 1973 - Proposal for the standardization of the presentation of annual events, especially of migrating data. Auspicium, 5: 49-59.

BirdLife International, 2015 - European Red List of Birds. Office for Official Publications of the European Communities, Luxembourg.

Boere G.C. \& Stroud D.A., 2006 - The flyway concept: what it is and what it isn't. In: Waterbirds around the world. Boere G.C., Galbraith C.A. \& Stroud D.A. (eds.). The Stationery Office, Edinburgh: 40-47.

Bordignon L. \& Corti W. 2003 - Tra cielo e acqua. Migratori in volo sul Pian di Spagna. Osservatorio Ornitologico Lodoletta e Consorzio Naturale Pian di Spagna-Lago di Mezzola. Tipolitografia di Borgosesia, Borgosesia.

Brichetti P. \& Fracasso G., 2004 - Ornitologia italiana. Vol. 2 - Tetraonidae - Scolopacidae. Alberto Perdisa Editore, Bologna.

Cairo E., Ferrario E., Bassi E., Caccia M. \& Rota R., 2001 L'avifauna della provincia di Bergamo: check-list aggiornata al 2001 e caratterizzazione fenologica. Rivista del Museo Civico di Scienze Naturali "E. Caffi”, Bergamo, 21: 47-85. 
Cardarelli E., Ranghetti L., Grieco C., Sindaco R., Longoni V., Bogliani G. \& Fasola M., 2013 - Cambiamenti colturali delle risaie italiane e disponibilità trofica per gli Ardeidi. XVII Convegno Italiano di Ornitologia. Trento, 11-15 Settembre 2013. Abstract 28.

Casale F., 2015 - Atlante degli Uccelli del Parco Lombardo della Valle del Ticino. Parco Lombardo della Valle del Ticino e Fondazione Lombardia per l'Ambiente.

Cucco M., Levi L., Maffei G. \& Pulcher C., 1996 - Atlante degli uccelli di Piemonte e Valle d'Aosta in inverno (1986-1992). Monografie 19. Museo Regionale di Scienze Naturali, Torino.

Delany S., Scott D., Dodman T. \& Stroud D. (eds). 2009 An Atlas of Wader Populations in Africa and Western Eurasia. Wetland International, Wageningen.

Della Toffola M., Alessandria G. \& Carpegna F., 2003 Nidificazione di Pettegola Tringa totanus in ambiente non alofilo in Italia. Avocetta, 27: 156.

Fasola M., Canova L. \& Saino N., 1996 - Rice fields support a large portion of herons breeding in the Mediterranean region. Colonial Waterbirds, 19: 129-134.

Fasola M. \& Ruiz X., 1996 - The value of rice fields as substitutes for natural wetlands for waterbirds in the Mediterranean region. Colonial Waterbirds, 19: 122128.

Fornasari L., Bottoni L., Massa R., Fasola M., Brichetti P. \& Vigorita V. (a cura di), 1992 - Atlante degli uccelli svernanti in Lombardia. Regione Lombardia e Università degli Studi di Milano, Milano.

Hötker H., Schradert S., Schwemmer P., Oberdiek N. \& Blew J., 2010 - Status, threats and conservation of birds in the German Wadden Sea. Technical Report. $N A B U$, Germany.

Ibañez C., Curcó A., Riera X., Ripoll I. \& Sánchez C., 2010 - Influence on birds of rice field management practices during the growing season: a review and an experiment. Waterbirds, 33: 167-180.

Lawler S.P., 2001 - Rice fields as temporary wetlands: a review. Israel Journal of Zoology, 47: 513-528.

Longoni V., 2010 - Rice fields and waterbirds in the Mediterranean region and the Middle East. Waterbirds, 33: 83-96.

Longoni V. \& Fasola M. 2012 - Censimento Annuale degli Uccelli Acquatici Svernanti in Lombardia. Resoconto 2011. Regione Lombardia, Milano.

Longoni V. \& Fasola M., 2013 - Censimento Annuale degli Uccelli Acquatici Svernanti in Lombardia. Resoconto 2013. Regione Lombardia, Milano.

Longoni V., Cardarelli E. \& Bogliani G., 2013 - Un esperimento di ricupero del valore naturalistico delle risaie: il progetto CORINAT. XVII Convegno Italiano di Ornitologia, Trento 11-15 Settembre 2013. Abstract 29.

Longoni V., Rubolini D., Pinoli G. \& Fasola M., 2014 Andamento delle popolazioni di uccelli acquatici svernanti in Lombardia 2002-2013. Rivista italiana di Ornitologia- Research in Ornithology, 84: 3-66.

Lourenço P.M. \& Piersma T., 2009 - Waterbird densities in South European rice fields as a function of rice management. Ibis, 151: 196-199.
Mingozzi T., 1982 - Resoconto ornitologico per la regione Piemonte-Valle d'Aosta da settembre 1979 all'agosto 1981. Rivista Piemontese di Storia Naturale, 3: 177-188.

Pillitteri Rosa D., Vigorita V. \& Fasola M., 2007 - Fenologia delle Specie di Uccelli Acquatici di Interesse Venatorio in Regione Lombardia - Relazione 20062007. Regione Lombardia, Milano.

Pirotta G., Redaelli G., Agostani G. \& Orsenigo F., 2009 L'avifauna della Palude di Brivio: analisi e proposte per un piano di gestione. Associazione Culturale " $\mathrm{Lu}$ igi Scanagatta", Varenna

Ranghetti L., Busetto L., Crema A., Fasola M., Cardarelli E. \& Boschetti M., 2015 - Stima delle variazioni della superficie allagata in risaia nel distretto lombardopiemontese tramite dati MODIS per il periodo 20002014. Conferenza ASITA 2015, Lecco, 29/09/2015-01 /10/2015: 913-916.

Scarton F., Baldin M. \& Valle R., 2009 - Distribuzione e consistenza della Pettegola Tringa totanus (Linnaeus, 1758) nidificante nelle barene della laguna aperta di Venezia. Anni 2001-2006. Bollettino del Museo civico di Storia naturale di Venezia, 59: 117-126.

Schelbert B., Fischer J., Gfeller S. \& Weggler M., 1995 Die Vogelwelt der Reussebene - Eine Entwicklungsgeschichte 1971-1993. Ala, Schweizerische Gesellschaft für Vogelkunde und Vogelschutz, Ornithologischer Beobachter.

Schneider-Jacoby M. \& Saveolic D., 2007 - Field Visit in the Natural Monument Velika Plaza, protected also as Coastal estate of Montenegro (Morsko Dobro) March 2007. Euronatur Report.

Serra L., Casini M., Della Toffola M., Magnani A., Meschini A. \& Tinarelli R., 1992 - Results of a survey on wader spring migration in Italy (March-May 1990). Wader Study Group Bulletin, 66: 54-60.

Spina F. \& Volponi S., 2008 - Atlante della Migrazione degli Uccelli in Italia. 1. Non - Passeriformi. Ministero dell'Ambiente e della Tutela del territorio e del Mare, Istituto Superiore per la Protezione e la Ricerca Ambientale (ISPRA). Tipografia CSR, Roma.

Tinarelli R., Giannella C. \& Melega L. (a cura di), 2010 Lo svernamento degli uccelli acquatici in Emilia-Romagna: 1994-2009. Regione Emilia-Romagna \& AsOER ONLUS. Tecnograf, Reggio-Emilia.

Toffoli R., 2008 - La migrazione dei Charadriiformes in Piemonte: stato attuale delle conoscenze ed indicazioni per la gestione e conservazione delle specie. Regione Piemonte, Osservatorio Regionale per la Fauna Selvatica.

Toral G.M. \& Figuerola J., 2010 - Unraveling the importance of rice fields for waterbird populations in Europe. Biodiversity and Conservation, 19: 2456-2469.

Tosi G. \& Zilio A. (eds.), 2002 - Conoscenza delle risorse ambientali della provincia di Varese-Progetto SIT-Fauna. Provincia di Varese, Settore politiche per l'Agricoltura e Gestione Faunistica.

Vaschetti G., Fasano S. \& Vaschetti B., 2001 - Fenologia migratoria del Piro piro culbianco Tringa ochropus in un'area del Piemonte. Avocetta 25: 73. 